\title{
Convex Optimization of Charging Infrastructure Design and Component Sizing of a Plug-in Series HEV Powertrain
}

\author{
Nikolce Murgovski* Lars Johannesson* Jonas Hellgren** \\ Bo Egardt* Jonas Sjöberg* \\ * Department of Signals and Systems, Chalmers University of \\ Technology, Gothenburg, Sweden (e-mail: \\ nikolce.murgovski@chalmers.se). \\ ** Volvo Technology, Gothenburg, Sweden.
}

\begin{abstract}
With the topic of plug-in HEV city buses, this paper studies the highly coupled optimization problem of finding the most cost efficient compromise between investing in onboard electric powertrain components and installing a charging infrastructure along the bus line. The paper describes how convex optimization can be used to find the optimal battery sizing for a series HEV with fixed engine and generator unit and a fixed charging infrastructure along the bus line. The novelty of the proposed optimization approach is that both the battery sizing and the energy management strategy are optimized simultaneously by solving a convex problem. In the optimization approach the power characteristics of the engine-generator unit are approximated by a convex, second order polynomial, and the convex battery model assumes quadratic losses. The paper also presents an example for a specific bus line, showing the dependence between the optimal battery sizing and the number of charging stations on the bus line.
\end{abstract}

Keywords: plug-in series powertrain, charging infrastructure, convex optimization

\section{INTRODUCTION}

Hybrid Electric Vehicles (HEVs) are widely regarded as one of the most promising means of achieving a nearterm reduction of emissions and energy consumption from the transportation sector. An HEV powertrain typically includes an internal combustion engine (ICE), one or more electric machines (EMs), and an energy buffer, which is usually a battery or a super capacitor. For a detailed overview on hybrid vehicles, see, eg, Guzzella and Sciarretta (2007).

The so-called plug-in HEVs (PHEVs) are equipped with a charging connector (typically an on-board charger), which allows the PHEVs to charge the electric buffer from the grid. PHEVs are designed to be charged either by the standard household electric power infrastructure or at stations installed on, eg parking lots, shopping malls, or other locations.

In recent years, PHEVs have been considered for use in public transportation by equipping high traffic bus lines with a charging infrastructure, KAIST (2009), AutoTram (2010), offering a flexible crossbreed between an HEV city bus and a tram. In KAIST (2009) the PHEV city bus is, while driving, inductively charged from underground cables that have been buried along sections of the bus line. In AutoTram (2010) the PHEV is equipped with a super capacitor which is charged at the bus stops through a docking station. Since the PHEVs are to be charged at relatively high power, the energy buffer makes it possibly to drive a significant part of the bus line on electric power even though the charging infrastructure might be sparsely distributed.

The cost optimal sizing of the energy buffer, ie power rating and energy capacity, of these PHEVs will be heavily dependent on the drive pattern and topography along the bus line as well as the charging infrastructure. In order to optimize the total cost efficiency of a PHEV public transportation system, it will therefore be crucial to find the best compromise between investing in onboard electric powertrain components and installing charging infrastructure along the bus line.

The solution to this optimization problem, however, depends not only on the city bus system configuration, the cost of the onboard electric components and the charging infrastructure, but also on changing factors such as fuel and electricity prices. Moreover, a complicating issue when evaluating $\mathrm{HEV}$ city buses is that the energy efficiency of the powertrain depends on how well adapted the energy management strategy is to the bus line, Johannesson (2009). For PHEV city buses the energy management strategy will decide the operating point of the ICE and thereby when and at what rate the energy buffer is to be discharged. When optimizing the PHEV public transportation system based on a dynamic model of the powertrain, a badly tuned energy management strategy might lead to a non-optimal sizing of the energy buffer and thereby a non-optimal charging infrastructure.

The conclusion is that there is a strong need to develop a systematic method that can optimize the cost efficiency of a PHEV public transportation system taking the energy 
management strategy as well as the powertrain component sizing and charging infrastructure into consideration. In the general case this optimization problem is a nonconvex, mixed integer problem, which means that there is little or no hope of finding a global optimum.

Nevertheless, in this paper it will be shown that if the PHEV is based on the so called series architecture, see Guzzella and Sciarretta (2007), and has a battery as energy buffer, the PHEV public transportation problem can be divided into smaller convex subproblems. These convex subproblems can be efficiently solved for a global optimum using generally available solvers, SeDuMi (Labit et al., 2002), SDPT3 (Toh et al., 2006). More specifically, the paper describes how convex optimization can be used to solve the subproblems of finding the optimal battery sizing for a series HEV with a fixed engine and generator unit and fixed charging infrastructure along the bus line. In the optimization approach the power characteristics of the engine-generator unit are approximated by a convex, second order polynomial, and the convex battery model assumes quadratic losses. The only heuristic choice in the optimization is the engine on/off operation.

Finally, the solution to the PHEV public transportation problem is found by solving the convex subproblems in two nested loops for: 1) all different sizes of the engine and generator unit, and 2) all possible distributions of charging stations on the bus line.

The paper is outlined as follows: the studied bus line and charging infrastructure are discussed in Section 2; the PHEV powertrain model is described in Section 3; the optimization problem is formulated in Section 4; the method for solving the problem is presented in Section 5; the convex modeling of the problem is given in Section 6; an example of battery sizing is given in Section 7 and the paper is ended with conslusion in Section 8.

\section{BUS LINE AND CHARGING INFRASTRUCTURE}

The studied bus line, see Fig. 1, is completely described by the reference velocity $v_{d e m}(t)$ and the road slope $\alpha(t)$ (Fig. 1 illustrates the road altitude instead of the slope). At each of the $n_{b s}$ bus stops along the line, it is possible to place a charging station to which the bus can dock on, or pick up electric power inductively, to charge its battery.

In the paper the number of charging stations along the line, $n_{c s}$, will be varied to study the effects on the optimal battery sizing. For each $n_{c s}$ the charging stations are distributed along the line with as equal distance to each other as possible.

At the $j$ th charging station the bus can be charged at $P_{j, \max }$ maximum power for the duration of the stop lasting $t_{j}$ seconds. Hence, the electric power that the whole charging infrastructure provides, can be expressed as

$$
\begin{aligned}
& P_{c s}\left(t, n_{c s}\right)=\left\{\begin{array}{ll}
P_{j, \max } & \text { for } t \in\left[t_{c s, j}, t_{c s, j}+t_{j}\right] \\
0 & \text { otherwise }
\end{array},\right. \\
& j=0, \ldots, n_{c s}, \quad n_{c s} \leq n_{b s}
\end{aligned}
$$

where $t_{c s, j}$ is the time when the bus arrives at the $j$ th charging station.
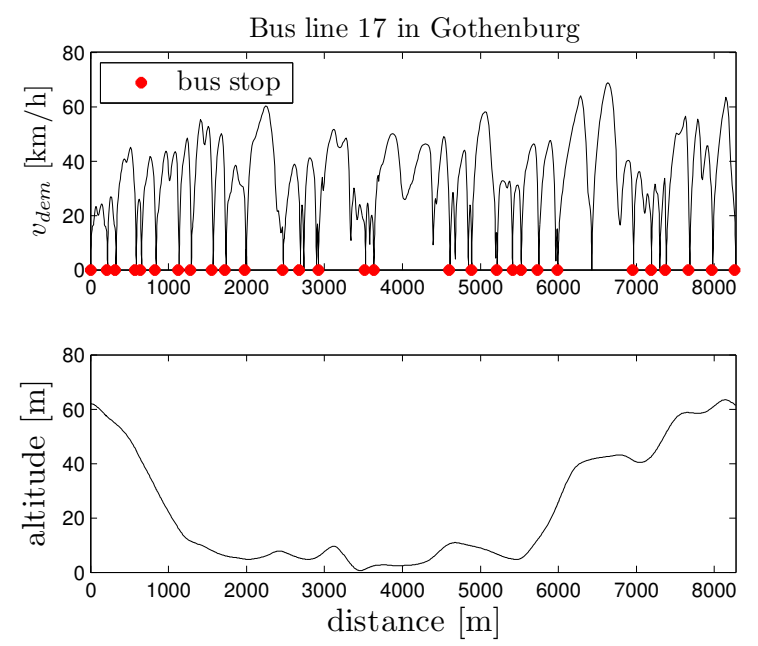

Fig. 1. Driving cycle. The top plot shows demanded velocity and position of the bus stops. The bottom plot shows the road altitude.

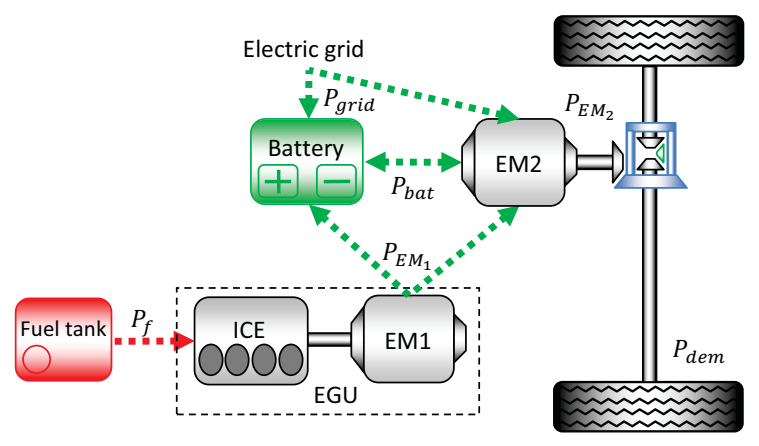

Fig. 2. Series PHEV powertrain model.

\section{PHEV POWERTRAIN MODEL}

The system architecture for the studied PHEV is shown in Fig. 2. The series powertrain includes an internal combustion engine (ICE), two electric machines (EMs) and a battery. The ICE together with the first electric machine (EM1), also known as the engine-generator unit (EGU), are used to supply electric energy to the second electric machine (EM2) and the battery. The vehicle is propelled entirely by EM2, which is mechanically coupled to the wheels.

The powertrain is modeled as a nonlinear inverse simulation model that satisfies the power balance equation

$$
\begin{aligned}
P_{\text {dem }}(t) & =\eta_{E M_{2}}(t) P_{E M_{2}}(t)+P_{b r k}(t) \\
P_{E M_{2}}(t) & =P_{b a t}(t)+P_{\text {grid }}(t)+P_{E M_{1}}(t) \\
P_{E M_{1}}(t) & =\eta_{E G U}(t) P_{f}(t)
\end{aligned}
$$

where $P_{\text {dem }}(t)$, detailed below in (5), is the power required to exactly follow the driving profile, $P_{b r k}(t)$ is braking power corresponding to the energy dissipated in the friction brakes, $P_{E M_{2}}(t)$ and $\eta_{E M_{2}}(t)$ are power and efficiency of EM2, $P_{b a t}(t)$ is the battery power, $P_{E M_{1}}(t)$ is the electric power delivered by EM1 and $\eta_{E G U}(t)$ is the EGU efficiency for a given fuel power $P_{f}(t)$. The electric power, $P_{\text {grid }}(t)$, that the PHEV takes from the grid can be expressed as

$$
P_{\text {grid }}(t)=x_{c s}(t) P_{c s}\left(t, n_{c s}\right), \quad x_{c s}(t) \in[0,1]
$$




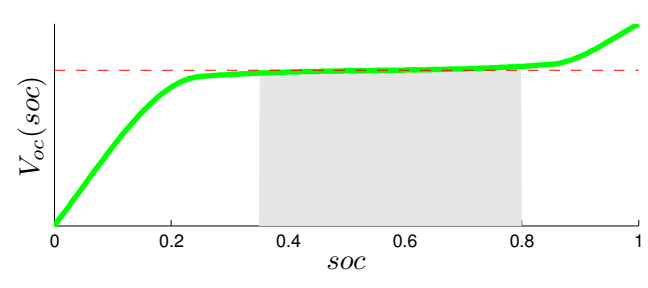

Fig. 3. Model of the battery open circuit voltage, as can be found in, eg, Guzzella and Sciarretta (2007). The solid line represents the original function, while the dashed line is the approximation. Good fit is expected in the shaded region.

where $x_{c s}(t)$ is a decision variable that determines at which rate the battery is to be charged when docked to a charging station.

The powertrain components are physically limited by

$$
\begin{aligned}
& P_{E M_{2}}(t) \in\left[P_{E M_{2}, \text { min }}, P_{E M_{2}, \text { max }}\right] \\
& P_{E M_{1}}(t) \in\left[0, P_{E M_{1}, \max }\right], \quad P_{b r k}(t) \leq 0 .
\end{aligned}
$$

The demanded power, $P_{d e m}(t)$, is uniquely determined by the driving cycle and the longitudinal dynamics of the vehicle, modeled as point mass, as,

$$
\begin{aligned}
P_{d e m}(t) & =\left(m \dot{v}_{d e m}(t)+\frac{\rho_{\text {air }}}{2} A_{f} c_{d} v_{d e m}^{2}(t)\right. \\
& \left.+m g c_{r} \cos \alpha(t)+m g \sin \alpha(t)\right) v_{d e m}(t)
\end{aligned}
$$

where $m$ is the vehicle mass, $A_{f}$ is the frontal area, $c_{d}$ is the drag coefficient, $c_{r}$ is the rolling resistance coefficient, $\rho_{\text {air }}$ is the air density and $g$ is the gravitational acceleration.

The EM2 efficiency is given by

$$
\eta_{E M_{2}}(t)= \begin{cases}\eta_{d} & \text { if } P_{d e m}(t)>0 \\ 1 / \eta_{c} & \text { otherwise }\end{cases}
$$

where $\eta_{c}$ is the charging and $\eta_{d}$ is the discharging efficiency.

The battery system consists of $n_{p}$ strings connected in parallel, where each string consists of $n_{s}$ identical battery cells in series. The power of the battery pack, modeling the cells as simple resistive circuits, Guzzella and Sciarretta (2007), can be computed as

$$
P_{b a t}(t)=\left(V_{o c}-R i(t)\right) i(t) n_{b c}, \quad n_{b c}=n_{s} n_{p}
$$

where $i(t)$ is the current in each of the strings, $V_{o c}$ is the open circuit voltage of the battery cell, $R$ is the cell resistance and $n_{b c}$ is the total number of cells.

The state of charge (SOC) of the battery cell is defined as

$$
\operatorname{soc}(t)=\operatorname{soc}_{0}-\frac{1}{Q} \int_{t_{0}}^{t} i(t) d t
$$

where $Q$ denotes the battery cell capacity and $s o c_{0}$ is the SOC at the initial time $t_{0}$. The SOC and cell current are limited according to

$$
\operatorname{soc}(t) \in\left[s o c_{\min }, s o c_{\max }\right], \quad i(t) \in\left[i_{\min }, i_{\max }\right]
$$

where the limit on the allowed SOC values is imposed to avoid excessive wear of the battery. The parameters $V_{o c}$ and $R$ are independent of $\operatorname{soc}(t)$, which is a valid assumption as long as $\operatorname{soc}(t)$ is kept within 35 to 80 percent charge, see Fig. 3.

A simple static model is used for the EGU, Neuman et al. (2008), where $\eta_{E G U}$ is efficiency curve modeled as

$$
\eta_{E G U}=\eta_{1}\left(1-e^{-\beta_{1}\left(P_{f}-P_{i d l e}\right)}\right)+\eta_{2} e^{-\beta_{2}\left(P_{f}-P_{f}^{*}\right)^{2}} .
$$

Due to internal friction, the efficiency approaches zero at the low power region $P_{i d l e}$. Then, as $P_{f}$ increases, the efficiency increases with rate $\beta_{1}$ to a value $\eta_{1}$. The maximum EGU efficiency is $\eta_{1}+\eta_{2}$ centered around $P_{f}^{*}$. The parameter $\beta_{2}$ determines the protuberance of the efficiency peak. Low $\beta_{2}$ value gives flatter curve around $P_{f}^{*}$, while higher $\beta_{2}$ gives a prominent peak, see the bottom row of Fig. 4. This modeling approach captures the essential EGU characteristics and compares reasonably well to manufacturer data.

\section{PROBLEM FORMULATION}

The objective of this study is to develop an efficient tool for solving the general PHEV public transportation problem of optimal tradeoff between investing in charging infrastructure and powertrain components. In this section, the general problem is formulated, although later, in Section 7, an example is given for a simpler problem where the optimization of the charging infrastructure has been removed from the problem.

The general cost function consists of cost for the consumed fuel and electricity plus additional costs $\Psi_{1}$ and $\Psi_{2}$

$$
\begin{aligned}
& J=w_{1} \int_{t_{0}}^{t_{f}} P_{f}(t) d t+w_{2} \int_{t_{0}}^{t_{f}} P_{g r i d}(t) d t+\Psi_{1}+\Psi_{2} \\
& \Psi_{1}=w_{3} m_{p}+w_{4} n_{b c} \\
& \Psi_{2}=w_{5}\left(n_{c s}\right)+w_{6}\left(P_{E M_{1}, \text { max }}\right)+w_{7}\left(P_{E M_{2}, \text { max }}\right)
\end{aligned}
$$

where $t_{f}$ is the final time and $w_{1}, \ldots, w_{7}$ transform the respective cost terms into money. The cost $\Psi_{2}$ is, in general, a nonlinear, non-convex function of the number of charging stations, $n_{c s}$, and the maximum EGU and EM2 power, $P_{E M_{1}, \max }, P_{E M_{2}, \max }$.

The cost $\Psi_{1}$ can be any convex function of the number of battery cells $n_{b c}$ and the maximum payload (passengers onboard) $m_{p}$, but here, for simplicity, it is assumed to be linear. These variables affect the vehicle mass, ie the power demand which can be expressed as

$$
\begin{aligned}
& P_{d e m}(t)=P_{b}(t)+C(t)\left(n_{b c} m_{b c}+m_{p}\right) \\
& C(t)=\left(\dot{v}_{d e m}(t)+g c_{r} \cos \alpha(t)+g \sin \alpha(t)\right) v_{d e m}(t)
\end{aligned}
$$

where $P_{b}(t)$ is baseline demanded power for the powertrain with fixed EGU and EM2 and no battery and passengers onboard.

The penalty coefficients for fuel, electricity and payload are computed as

$$
w_{1}=\frac{c_{1}}{Q_{f}}, \quad w_{2}=\frac{c_{2}}{3600}, \quad w_{3}=-\frac{c_{3}}{m_{p, a v g}}
$$

where $Q_{f}$ is the energy content of the fuel, $c_{1}$ is the fuel price, $c_{2}$ is the electricity price in [currency $\left./ \mathrm{kWh}\right]$ and $c_{3}$ and $m_{p, a v g}$ are the typical total income and passengers' mass on the bus line. The costs for the charging stations and the powertrain components are computed by assuming that the payment for the components' cost is equally divided in $n_{j}$ years with $p_{j}$ percent yearly interest rate. This yields

$$
\begin{aligned}
w_{j} & =\frac{c_{j}}{n_{j} s_{\text {avg }}}\left(1+\frac{p_{j}}{100} \frac{n_{j}+1}{2}\right) \int_{t_{0}}^{t_{f}} v_{d e m}(t) d t \\
j & =4, \ldots, 7
\end{aligned}
$$

where $s_{\text {avg }}$ is the average distance traveled by the vehicle in one year, $c_{4}$ and $c_{5}\left(n_{c s}\right)$ are the costs, in [currency], 
for the battery cell and the charging infrastructure and $c_{6}\left(P_{E M_{1}, \max }\right)$ and $c_{7}\left(P_{E M_{2}, \max }\right)$ are the costs, in [currency $/ \mathrm{kW}$ ] for the EGU and EM2.

The optimization is subject to the constraints (1)-(10). Additional possible constraints are starting at desired SOC value and preserving charge sustain operation

$$
\operatorname{soc}\left(t_{0}\right)=\operatorname{soc}_{0}, \quad \operatorname{soc}\left(t_{0}\right)=\operatorname{soc}\left(t_{f}\right) .
$$

The problem consists of six time varying optimization variables, $P_{E M_{1}}(t), P_{E M_{2}}(t), P_{b a t}(t), \operatorname{soc}(t), i(t)$ and $x_{c s}(t)$, and five time invariant variables, $n_{b c}, n_{c s}, P_{E M_{1}, \max }$, $P_{E M_{2}, \max }$ and $m_{p}$.

\section{OPTIMIZATION METHODOLOGY}

The problem described in Section 4 is both nonlinear and non-convex, containing a mix of integer and continuous variables. Often the best strategy of attacking such problem is to split it, if possible, in convex and non-convex part ("divide and conquer"), Boyd and Vandenberghe (2004). In Section 6 it is shown that with some reasonable approximations, part of the problem can be represented as convex. The global optimum is then found by solving several convex problems in each iteration of two nested loops:

(1) Define desired vehicle performance by deciding the driving cycle, as in Fig. 1.

(2) Loop through all given sizes of EGU and EM2.

(3) For each EGU and EM2, loop through all possible distributions of charging stations on the bus line.

(4) In each iteration solve the convex problem that will be explained later in Section 6 .

The convex problem is solved using CVX, a package for specifying and solving convex programs, Grant and Boyd (2010).

\section{DISCIPLINED CONVEX MODELING}

In this section the problem formulated in Section 4 is approximated as convex by using the methodology of disciplined convex programming as proposed by Boyd and Vandenberghe (2004).

\subsection{Convex battery model}

The battery constraint ( 7$)$ is not convex, since it involves a product of two variables, $n_{b c}$ and $i(t)$. The constraint can however be reformulated as convex in two steps. First, by performing the following variable changes

$$
\tilde{i}(t)=n_{b c} i(t), \quad s \tilde{o} c(t)=n_{b c} \operatorname{soc}(t)
$$

the constraints $(7),(8),(9)$ and (15) can be rewritten as

$$
\begin{aligned}
& P_{b a t}(t)=V_{o c} \tilde{i}(t)-P_{l o s s}(t) \\
& P_{l o s s}(t)=R \frac{\tilde{i}^{2}(t)}{n_{b c}} \\
& s \tilde{O} c(t)=n_{b c} s o c_{0}-\frac{1}{Q} \int_{t_{0}}^{t} \tilde{i}(t) d t \\
& s \tilde{O} c(t) \in\left[\operatorname{soc}_{\min }, s o c_{\max }\right] n_{b c} \\
& \tilde{i}(t) \in\left[i_{\text {min }}, i_{\text {max }}\right] n_{b c} \\
& s \tilde{O} c\left(t_{0}\right)=\operatorname{soc}_{0} n_{b c}, \quad s \tilde{O} c\left(t_{0}\right)=s \tilde{O} c\left(t_{f}\right)
\end{aligned}
$$



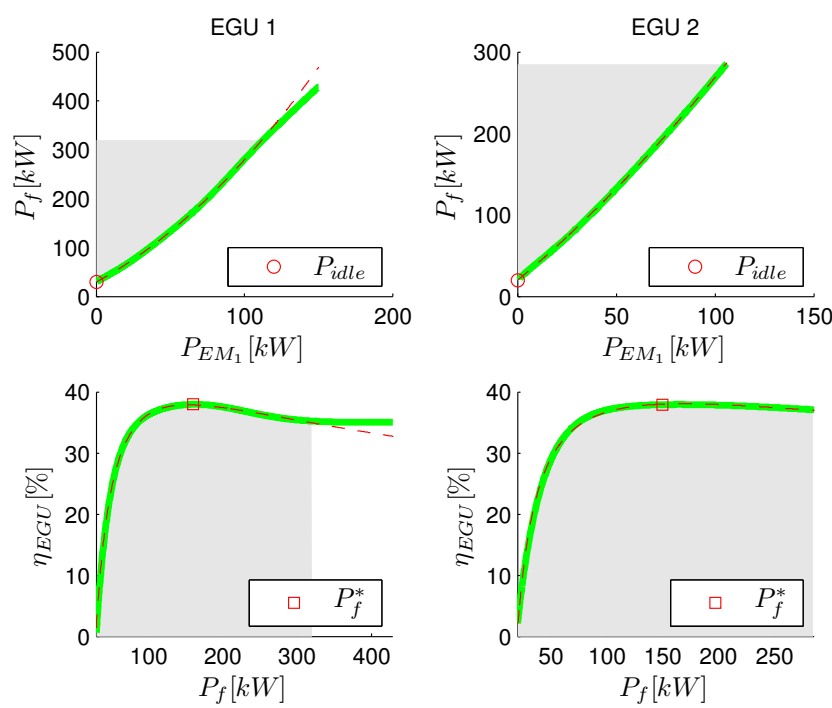

Fig. 4. Model of two different EGUs. The first EGU in the two left plots has high $\beta_{2}$ value, while the second EGU in the two right plots has low $\beta_{2}$ value. The solid line represents the original function, while the dashed line is the approximation. The shaded region shows the interval $P_{f} \in\left[P_{f, l o w}, P_{f, h i g h}\right]$ in which good fit is to be expected.

$$
\begin{aligned}
& \bar{P}_{f}(t)=\left(a_{0}+a_{1} \tilde{P}_{E M_{1}}(t)+a_{2} \tilde{P}_{E M_{1}}^{2}(t)\right) e_{o n}(t) \\
& P_{E M_{1}}(t)=\tilde{P}_{E M_{1}}(t) e_{o n}(t)
\end{aligned}
$$

where $e_{o n}(t)$ is a binary variable, 0 or 1 , that governs the ICE on/off operation and ensures that no electric power is generated by EM1 when the ICE is off. However, (22) is not convex, which is obviously visible in the top row of Fig. 4, when the operating point at the origin is included. Convexity can be preserved by using a heuristic decision on the value of $e_{o n}(t)$. The heuristics may define $e_{\text {on }}(t)$ along the bus line as any function of the speed profile, topography, distance to a charging station, time etc, but the parameters governing the heuristics must be kept constant during the convex optimization.

\subsection{Convex EM2 model}

The EM2 model (6) can be represented as convex by relaxing (6) in two inequality constraints

$$
\begin{aligned}
P_{E M 2}(t) & \geq \frac{1}{\eta_{d}} P_{d e m}(t) \\
P_{E M 2}(t) & \geq \eta_{c} P_{d e m}(t)
\end{aligned}
$$

where $P_{\text {dem }}(t)$ is an affine function of the vehicle mass, ie the number of battery cells and payload. Then, from (2), the braking power of the friction brakes can be expressed as

$$
P_{b r k}(t)= \begin{cases}0 & \text { if } P_{\text {dem }}(t) \geq 0 \\ P_{\text {dem }}(t)-P_{E M_{2}}(t) / \eta_{c} & \text { otherwise. }\end{cases}
$$

With similar reasoning as in Section 6.1 it can be concluded that the optimal solution of the relaxed problem will satisfy one of the constraints (23) and (24) with equality at each time instant. Hence, it will be optimal solution to the original problem as well.
Table 1. Convex problem.

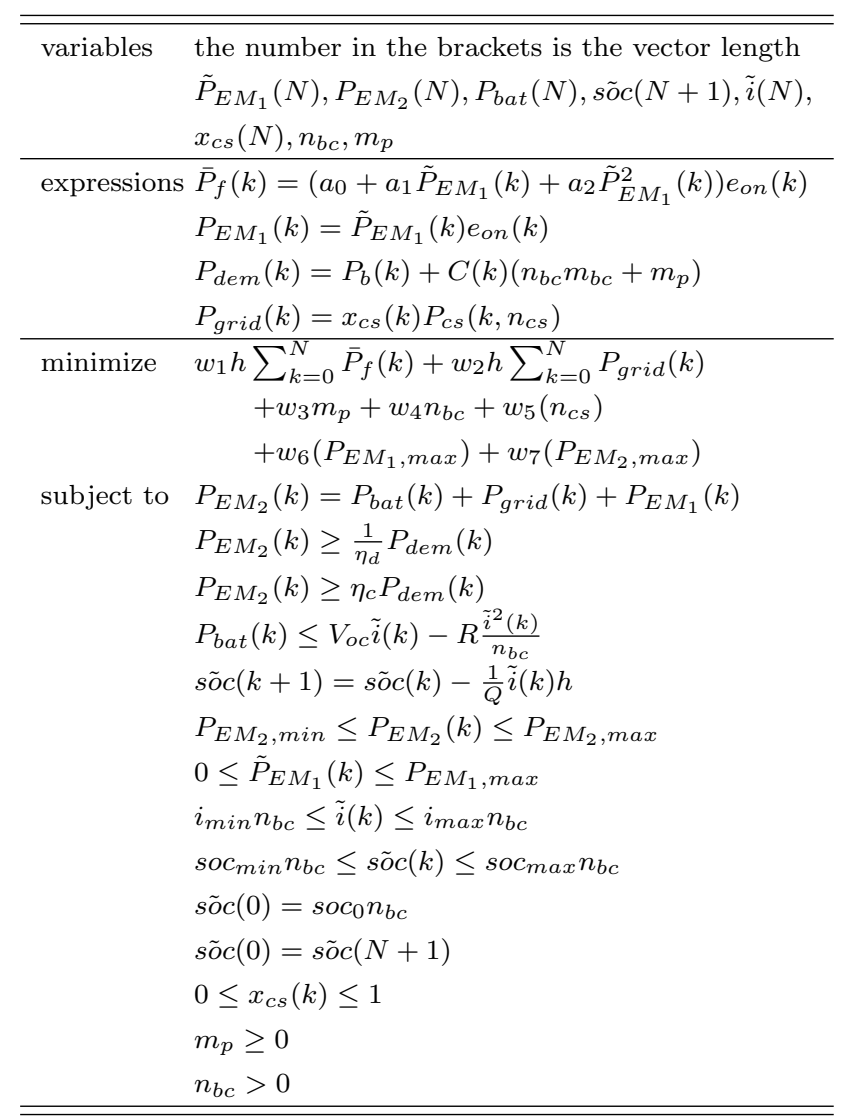

\subsection{The convex problem}

Using the convex models of the powertrain components, the final convex problem can be summarized as in Table 1. The convex problem is solved in each iteration of the two nested loops, as explained in Section 5, after it has been rescaled and discretized with zero order hold. For a chosen sampling time $h$, the discretization will give $k=1, \ldots, N$ time instances. Hence each time dependent variable in the convex problem is actually a vector of $N=t_{f} / h+1$ variables. Note that the same variable notation, see Table 1 , is used for the discrete variables as was used for the corresponding continuous variables.

\section{EXAMPLE: BATTERY SIZING}

This section gives an example of optimal battery sizing of a series PHEV bus for a fixed Level 2 and Level 3 infrastructure, Wiederer and Philip (2010). The charging stations on the bus line, see Fig. 1, are all assumed to provide the same magnitude of power, which is $10 \mathrm{~kW}$ for Level 2 and $100 \mathrm{~kW}$ for Level 3 stations. It is also assumed, for simplicity, that the vehicle charging interval is equal at each station and two cases for the interval length are considered, $10 \mathrm{~s}$ and $20 \mathrm{~s}$. The power of the EGU is $100 \mathrm{~kW}$ and the efficiency curve is as in the right plot of Fig. 4. The battery type is Lithium-ion with energy density of $41 \mathrm{Wh} / \mathrm{kg}$ and power density of $1.2 \mathrm{~kW} / \mathrm{kg}$. The initial battery state is free, but charge sustaining operation is maintained, assuming that immediately after the final stop, the bus may start the route in opposite direction. The battery cost of $235 € / \mathrm{kWh}$ ought to be paid in 2 years 
Table 2. Parameter values.

\begin{tabular}{l|l||l|l}
\hline Parameter & Value & Parameter & Value \\
\hline$m_{b}$ & $11800 \mathrm{~kg}$ & $P_{E M_{2}, \max }$ & $170 \mathrm{~kW}$ \\
$A_{f}$ & $8.05 \mathrm{~m}^{2}$ & $P_{E M_{2}, \text { min }}$ & $-170 \mathrm{~kW}$ \\
$c_{d}$ & 0.79 & $\eta_{d}$ & 0.9 \\
$c_{r}$ & 0.009 & $\eta_{c}$ & 0.5 \\
$V_{o c}$ & $3.6 \mathrm{~V}$ & $\rho_{\text {air }}$ & $1.293 \mathrm{~kg} / \mathrm{m}^{3}$ \\
$R$ & $1 \mathrm{~m} \Omega$ & $g$ & $9.82 \mathrm{~m} / \mathrm{s}^{2}$ \\
$m_{b c}$ & $0.85 \mathrm{~kg}$ & soc $_{\min }$ & 0.4 \\
$h$ & $2 \mathrm{~s}$ & soc $_{\max }$ & 0.6 \\
$c_{1}$ & $1.34 € / 1$ & $s_{\text {avg }}$ & $50000 \mathrm{~km}$ \\
$c_{2}$ & $0.1 € / \mathrm{kWh}$ & $T$ & $6 \mathrm{~s}$ \\
\hline
\end{tabular}

with $5 \%$ yearly interest rate. The values for the rest of the parameters are given in Table 2, where the vehicle mass $m_{b}=m-n_{b c} m_{b c}-m_{p}$ corresponds to the powertrain without the battery and passengers onboard. The costs for the EGU, EM2, the charging infrastructure, the power electronics and the payload are excluded from the problem, ie $w_{3}=w_{5}=w_{6}=w_{7}=0$.

\subsection{Heuristic ICE on/off decision}

The ICE on/off decision is based on the demanded vehicle power

$$
e_{o n}(t)= \begin{cases}1 & \text { if } \frac{1}{T} \int_{t}^{t+T} P_{b}(t) d t \geq P_{o n}^{*} \\ 0 & \text { otherwise }\end{cases}
$$

where the ICE is turned on when the mean of the demanded power from the baseline powertrain in the next $T$ seconds is higher than $P_{o n}^{*}$. The power $P_{o n}^{*}$ is the optimal threshold that results in a feasible solution and minimizes the cost (11). It is obtained by exhaustive search, ie, by first gridding the power, $\tilde{P}_{o n, i} \in\left[0 ; \eta_{d} P_{E M_{2}, \max }\right]$, and then solving the convex problem for each gridded value $\tilde{P}_{o n, i}$ as a switching threshold. This will insert an additional nested loop in the algorithm described in Section 5.

\subsection{Optimization results}

The optimization results, given in Fig. 5, show the optimal number of battery cells as a function of the number of charging stations on the bus line. In the case of Level 2 charging stations, see the left plot of Fig. 5, the optimal battery size increases with the number of stations. This is because the total cost for battery and the used electricity is lower than the cost for the used diesel fuel, hence it is optimal to increase the battery size with the number of charging stations to make room for storing the relatively cheap electric energy from the grid. The optimal solution, in this case, resulted in charging the battery with full rate during the whole charging interval for all distributions of charging stations.

Similar results are obtained for the case of Level 3 charging stations with $10 \mathrm{~s}$ charging interval per station, see the right plot of Fig. 5. However, after the charging interval had been increased to $20 \mathrm{~s}$, it was found that with 19 stations, the quantity of electric energy taken from the grid is large enough for the bus to drive the route entirely as electric vehicle (EV). As the number of charging stations increases even further, the optimal battery size starts to decrease, as smaller battery can still achieve EV operation.
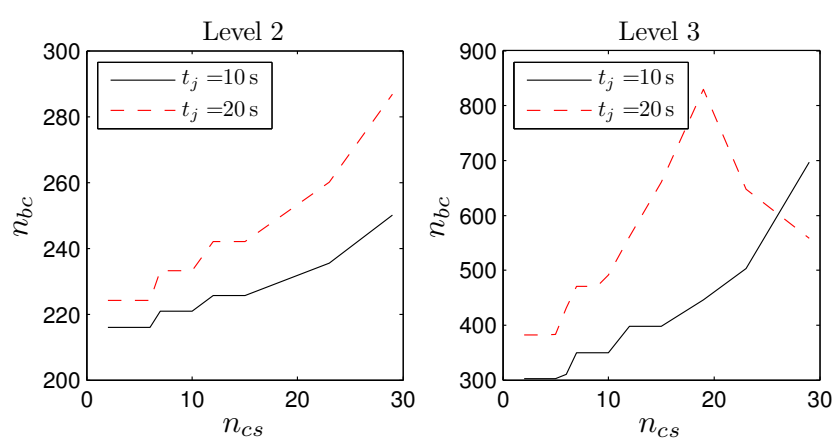

Fig. 5. Optimal battery size per number of charging stations.

\section{CONCLUSION}

This paper presented a method for optimal sizing of series PHEV powertrains and charging infrastructure. The optimization problem is approximated as a nonlinear convex problem, using heuristics only for the ICE on/off operation. This study also showed an example of powertrain sizing for which the complete code was provided as it can be implemented in CVX.

Further developments can be made by using smarter decision on then ICE on/off heuristics, improved cost function that accounts for battery wear and possibility to work with more detailed models of the powertrain components, eg nonliner, non-convex EM2 model.

\section{REFERENCES}

On-line electric vehicle. Technical report, Korea Advanced Institute of Science and Technology (KAIST), 2009.

AutoTram: Transport system of the future. Technical report, Fraunhofer Institute for Transportation and Infrastructure Systems IVI, 2010.

S. Boyd and L. Vandenberghe. Convex Optimization. Cambridge University Press, 2004.

M. Grant and S. Boyd. CVX: Matlab software for disciplined convex programming, version 1.21 . http //cvxr.com/cvx, May 2010.

L. Guzzella and A. Sciarretta. Vehicle propulsion systems, introduction to modeling and optimization. Springer, 2nd edition, 2007.

L. Johannesson. Predictive Control of Hybrid Electric Vehicles on Prescribed Routes. PhD thesis, Chalmers University of Technology, Göteborg, Sweden, 2009.

Y. Labit, D. Peaucelle, and D. Henrion. SeDuMi interface 1.02: a tool for solving lmi problems with SeDuMi. IEEE International Symposium on Computer Aided Control System Design Proceedings, pages 272-277, September 2002.

M. Neuman, H. Sandberg, B. Wahlberg, and A. Folkesson. Modelling and control of series hevs including resistive losses and varying engine efficiency. SAE International, 2008.

K. C. Toh, R. H. Tütüncü, and M. J. Todd. On the implementation and usage of SDPT3 - a matlab software package for semidefinite-quadratic-linear programming, version 4.0. July 2006 .

A. Wiederer and R. Philip. Policy options for electric vehicle charging infrastructure in $\mathrm{c} 40$ cities. Technical report, 2010. 